\title{
Calculating the $x$ Dependence of Hadron Parton Distribution Functions
}

\section{Huey-Wen Lin*}

Department of Physics, University of Washington, Seattle, WA 98195-1560

\begin{abstract}
I present a first direct lattice-QCD calculation of the $x$ dependence of hadron structure functions. By taking a hadron with a large momentum boost, we are able to connect light-cone quantities to lattice-QCD nonlocal but time-independent matrix elements. Since the largest attainable momentum is limited, we correct for the sizable leading momentum dependence systematically. I present a first study of the nucleon quark density, helicity and transversity distributions, and pion distribution amplitudes on the lattice; I demonstrate this can be done using today's computer resources and directly on the physical pion mass next.
\end{abstract}

31st International Symposium on Lattice Field Theory LATTICE 2013

July 29 - August 3, 2013

Mainz, Germany

*NT@UW-14-02. This is a progress report on work done in collaboration with Jiunn-Wei Chen, Saul D. Cohen and Xiangdong Ji. We thank MILC collaboration for sharing the lattices used to perform this study. The LQCD calculations were performed using the Chroma software suite [1] on Hyak clusters at the University of Washington managed by the UW Information Technology, using hardware awarded by NSF grant PHY-09227700. The work of HWL is supported by DOE grant DE-FG02-97ER4014. 
Studying the structure of hadrons, such as nucleons and pions, is not only important to understanding the strong interactions of quarks and gluons, but also to improving the precision of new-physics searches. Since a broad class of experiments, including the LHC and dark-matter detection, require interactions with nucleons, the mission to probe femtoscale physics is also essential for disentangling Standard-Model (SM) contributions from potential new physics. These $\mathrm{SM}$ backgrounds require parton distribution functions (PDFs) as inputs. For example, the recent IceCube observation of an excess in $\mathrm{PeV}$ neutrinos does not necessarily require new physics, since it is within uncertainty of the SM contribution due to the uncertainties in the parton distribution functions [2]. However, due to the difficulty of working in the strong-interaction regime, after decades of experiments and theoretical efforts, there still remain many unknowns. Experiments, such as deep inelastic scattering (DIS) of electrons off nucleons generate cross sections containing information about nucleon structure, but they are not complete. Where theory and experiment are lacking, assumptions are often imposed, such as charge symmetry or particular forms for the strange and sea (antiquark) distributions. Although the valence-quark distributions are consistent among most of the analyses in the intermediate- $x$ region due to the constraints of experimental data, at large and small $x$, there are significant differences. Similar uncertainty applies to many quantities that are less constrained by experiment, such as antiquarks, polarized quarks, strange quarks and gluons (see Ref. [3] for a review). In the transversity case, there have been only a few attempts to extract valence-quark information, and many more assumptions must be used, compared with the unpolarized and longitudinally polarized cases. Although there exist a variety of model approaches to treat the structure functions, a nonperturbative approach from first principles, such as lattice QCD (LQCD), provides hope to resolve many of the outstanding theoretical disagreements and provide information in regions that are unknown or difficult to observe in experiments.

Probing hadron structure with lattice QCD has been limited to only the first few moments, due to complications arising from the breaking of rotational symmetry by the discretized Euclidean spacetime. The nonzero lattice spacing breaks the symmetry group of Euclidean spacetime from $O(4)$ to the discrete hypercubic subgroup $H(4)$. Due to the reduced symmetry, the required operators are more complicated and sometimes mix with each other. This is treatable but complicated. For moments beyond the third, no operators are free from divergence issues. This applies to other structure functions, as well as generalized parton distributions (GPDs).

Despite these problems, since the magnitudes of the higher moments drop quickly, the moment expansion has been considered a reasonable approximation for many years. Due to the complications mentioned above, LQCD calculations have not been extended beyond the lowest few moments. However, upcoming precision experiments raise the question of whether LQCD precision needs to be improved and if we need moments beyond $n=3$. Furthermore, experimental measurements focus on specific regions of $x$; this means that moment expansions are difficult to compare with experimental data. Multiple ideas have been proposed to improve the scope and precision of LQCD structure calculations, but the small signals result in poor signal-to-noise ratios which make it harder to do a precision calculation. Rather, improvements have mainly come with respect to the systematics, using lighter pion masses and adding dynamical up/down, strange and even charm degrees of freedom in the sea.

With increasing computational resources becoming available to the lattice-QCD community, it is possible that one day in the future, we will be able to overcome the signal-to-noise problem 
in higher-moment contributions and gain information on the moments beyond $3[4,5,6]$. One can model the $x$-dependence and reproduce the all calculated lattice moments to gain information on the $x$ dependence [7]. However, this will only give the combinations of the total or difference of quark and antiquark contributions rather than individual contributions. Experiments such as E665 at FNAL can probe nucleon sea flavor asymmetry, meaning that lattice QCD will be excluded if it can only apply traditional moment calculations. Similarly, STAR at RHIC is probing the polarized (anti)quark structure of nucleon. A new approach is needed to extend the reach of latticeQCD calculations of PDFs, to find a computationally friendly method to catch up with ongoing experimental efforts.

The recent paper by X. Ji [8] proposed a much more straightforward way of calculating the full $x$ dependence of the distribution for PDFs and GPDs (and other quantities) without dealing with many moments nor requiring enormous computational resources to achieve. In short, we take an operator containing an integral over the gluonic field strength and boost the nucleon momentum toward the speed of light, tilting the spacelike line segment toward the light-cone direction. The time-independent, nonlocal (in space) correlators at finite $P_{z}$ can be directly evaluated on the lattice. For example, the quark unpolarized distribution can be calculated via

$$
q_{\text {lat }}\left(x, \mu, P_{z}\right)=\int \frac{d z}{4 \pi} e^{i z k} \times\left\langle\vec{P}\left|\bar{\psi}(z) \gamma_{z}\left(\prod_{n} U_{z}(n \hat{z})\right) \psi(0)\right| \vec{P}\right\rangle
$$

where $U_{\mu}$ is a discrete gauge link in the $\mu$ direction, $x=k / P_{z}, \mu$ is the renormalization scale and $\vec{P}$ is the momentum of the hadron, taken such that $P_{z} \rightarrow \infty$. Since no amount of boost will take the nucleon exactly onto the light-cone, there remain corrections power-suppressed by $P_{z}$ as $O\left(M_{H}^{2} / P_{z}^{2}, \Lambda_{\mathrm{QCD}}^{2} / P_{z}^{2}\right)$ (where $M_{H}$ is hadron mass). The same idea can be straightforwardly applied to helicity $\Delta q(x, \mu)$ and transversity $\delta q(x, \mu)$, using $\gamma_{z} \gamma_{5}$ and $\sigma_{x y}$, respectively.

To demonstrate the feasibility of Ji's idea, we carried out an exploratory study using a small local cluster at University of Washington. We use clover valence fermions on an ensemble of gauge configurations with lattice spacing $a \approx 0.12 \mathrm{fm}$, box size $L \approx 3 \mathrm{fm}$ and pion mass $M_{\pi} \approx 310 \mathrm{MeV}$ with $N_{f}=2+1+1$ flavors of highly improved staggered quarks (HISQ), generated by MILC Collaboration[9], and we apply hypercubic (HYP) smearing to the gauge links. HYP smearing has been shown to significantly improve the discretization effects on operators and shift their corresponding renormalizations toward their tree-level values (near 1 for quark bilinear operators). Using a volume large enough such that $M_{\pi} L \approx 4.5$ keeps the finite-volume correction within a reasonable range for current lattice-QCD calculations of nucleon matrix elements. The results shown in this work are from a study including correlators calculated from 3 source locations on 449 configurations. To control the systematics due to contamination of ground-state quantities by the nearby excited state, a simultaneous fit of the nucleon matrix element correlator is performed with source-sink nucleon separations 0.96 and $1.2 \mathrm{fm}$; the detailed analysis procedure is described in Ref. [10].

We study isovector nucleon structure functions: the quark density, helicity and transversity distributions. The extracted matrix elements for various $z$ for quark density distribution are shown in Fig. 1. The different colors indicate $P_{z}$ (in units of $2 \pi / L$ ) 1 (red), 2 (green), 3 (cyan), and noise gets worse as we boost the nucleon to higher momentum. In all three cases, the bare lattice 

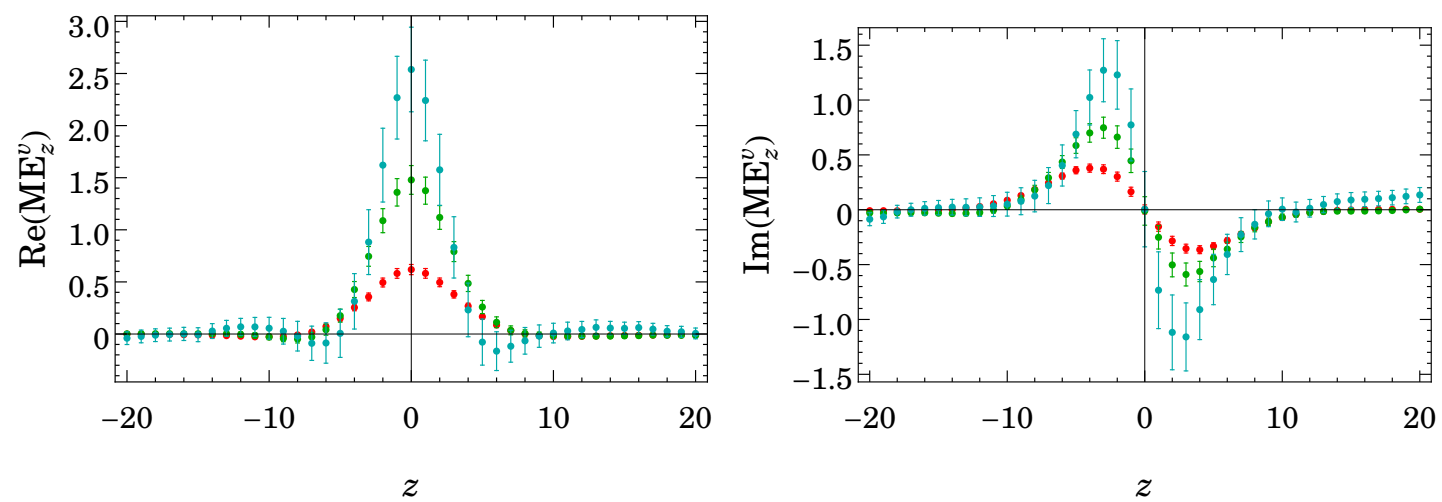

Figure 1: The real (left) and imaginary (right) parts of the nonlocal isovector matrix element needed for the PDF as functions of the length $z$ of the gauge connection between the quark and antiquark fields in the current insertion. The different colors indicate $P_{z}$ (in units of $2 \pi / L$ ) 1 (red), 2 (green), 3 (cyan).

nucleon matrix elements vanish around 10-12 links. The real (imaginary) matrix elements are (anti-)symmetric around $z=0$.

We then Fourier transform the $z$ coordinate into momentum $k$ to obtain $\tilde{q}_{\text {lat }}$. In the infinite$P_{z}$ limit, $x$ is the ratio of $k$ to $P_{z}$. Figure 2 shows our preliminary results on all three nucleon distributions (normalized by the vector/axial/tensor charge obtained from the $0^{\text {th }}$ moment integral; the normalized isovector nucleon charges at $4 \mathrm{GeV}^{2}$ can be found in Ref. [10]). We hope this would reduce the systematic uncertainty arising from the matching, renormalization, and other sources such as finite-volume effects and lattice discretization. We see that our lattice-QCD result has nonzero values at $x=1$. This is not hard to understand: when not in the infinite-momentum frame, constituents of the nucleon can carry more momentum than the nucleon. As we can see, the structure gets narrower as we increase $P_{z}$; this is a sign that we are converging onto the light-cone limit.

There are multiple crosschecks that can be done for these quantities, which is helpful since they are calculated for the first time without recourse to the literature for comparison. For example, the area under the distribution should be conserved for various $P_{z}$ (simply due to charge conservation) within statistical errors; indeed, this is what we find. We can take these distributions and integrate to find the lowest moments, which will give the vector, axial, and tensor charges. Using the OPE method mentioned in the introduction, this can be easily done and compared with results obtained from these distributions. We find that they indeed agree within the statistical errors. Similarly, we can check the second-lowest moments, and they agree well with traditional methods within errors.

In contrast to the traditional lattice method, we can obtain separate quark and antiquark distributions. What can we learn about the nucleon sea flavor asymmetry from our current calculation? Theoretical understanding of the nucleon sea has mostly come from nucleon models, such as the meson-cloud model, chiral-quark soliton model and others. Even though there are global analyses trying to take advantage of all available data sets and combine experiments with diverse kinematics, many still require assumptions for the sea flavor asymmetry, especially in the longitudinally and 
transversely polarized cases. (For example, see Ref. [3] for a review.) As shown in Figs. 2, we can clearly see the quark and antiquark flavor asymmetry in nucleon easily.

Note that we have not yet applied the finite-momentum corrections, which come in at the order of $\Lambda_{\mathrm{QCD}}^{2} / P_{z}^{2}, M_{N}^{2} / P_{z}^{2}$ and $O\left(\alpha_{s}\right)$ in this proceeding. The mass correction would be significant for lower-momentum distributions in the nucleon, and the correction to all orders of $M_{N}^{2} / P_{z}^{2}$ is underway. At the moment, we will take the $P_{z}=3$ as the infinite-momentum result to compare the relative sign and magnitude of the flavor sea asymmetry.

A large asymmetry in the up and down antiquarks in the nucleon was first observed by the New Muon Collaboration (NMC) as a violation of the Gottfried sum rule through the cross-section ratio for DIS of muons from hydrogen and deuterium [11]. Their data indicated that there are more down than up antiquarks in the proton, a result that was later confirmed by other experiments using different processes, such as Drell-Yan at E665 [12] and E866/NuSea [13]. Naively, one assumes the quark-antiquark pairs produced perturbatively from gluons should be flavor symmetric: $\bar{u} \approx \bar{d}$. Thus, a large observed asymmetry suggests a nonperturbative origin for the effect. The mesoncloud model tries to explain the quark sea as being generated by cloud of mesons, and the chiralquark soliton model treats quark degrees of freedom in a pion mean field. Both show asymmetries in the flavored antiquark distributions. Our $\int_{0}^{\infty} d x(\bar{d}(x)-\bar{u}(x))$ is $0.196(28)^{1}$, which is close to the experimental result obtained by NMC in their DIS measurement [11], 0.147(39) at $Q^{2}=4 \mathrm{GeV}^{2}$ and by HERMES in their semi-inclusive DIS (SIDIS) result, 0.16(3) at $Q^{2}=2.3 \mathrm{GeV}^{2}$ [14].

The nucleon polarized (helicity) sea asymmetry can be measured experimentally through polarized SIDIS at HERMES or $W$ production at RHIC. STAR's measurement of the longitudinal single-spin asymmetry and parity-violating $W$ production reveal information on the quark and antiquark polarizations. In their earlier Run 9, they obtained results that are consistent with theory evaluations (such as DSSV) using data from polarized DIS. However, the more recent 2012 run revealed a larger asymmetry [15] above DSSV, suggesting a large up antiquark polarization. Similar results are also observed by PHENIX. We note that even though the data at RHIC agrees with us for $\Delta \bar{u}>\Delta \bar{d}$, the $\int_{0}^{1} d x(\Delta \bar{u}(x)-\Delta \bar{d}(x))$ is much smaller, $\approx 0.07$; note that the renormalization scale is quite different $Q^{2}=10 \mathrm{GeV}^{2}$. HERMES's results at $Q^{2}=2.5 \mathrm{GeV}^{2}$ appear to have consistent sign, but it is not statistically well resolved from zero [16].

Our helicity asymmetry $\int_{0}^{\infty} d x(\Delta \bar{u}(x)-\Delta \bar{d}(x))$ is $0.294(13)$; our sign is consistent with a recent STAR and PHENIX runs [15], which also suggest a large up-antiquark polarization at $Q^{2}=10 \mathrm{GeV}^{2}$. Various meson-cloud models [17] yield a wide range of values from -0.7 to 0.12; Pauli-blocking models give 0.09-0.30; a chiral-quark soliton model combined with large- $N_{c}$ limit gives 0.31. A statistical model also suggests the magnitude (but opposite sign) of the flavor asymmetry to be about the same amount as the unpolarized; they estimate the polarized sea to be about 0.12 . We do observe a large polarized sea asymmetry, which is larger than the unpolarized sea contribution, as predicted as large- $N_{c}$ limit theory; this agrees with models based on large- $N_{c}$, such as chiral-quark soliton model. It would be interested to see how the distribution changes as we lower the quark mass, which is probably the dominant difference between the lattice and physical distributions. For more model estimations for the unpolarized and polarized sea asymmetry, we

\footnotetext{
${ }^{1}$ The sea-quark distribution can be read from the negative- $x$ contribution: $\bar{q}(x)=-q(-x), \delta \bar{q}(x)=-\delta q(-x)$, $\Delta \bar{q}(x)=\Delta q(-x)$.
} 

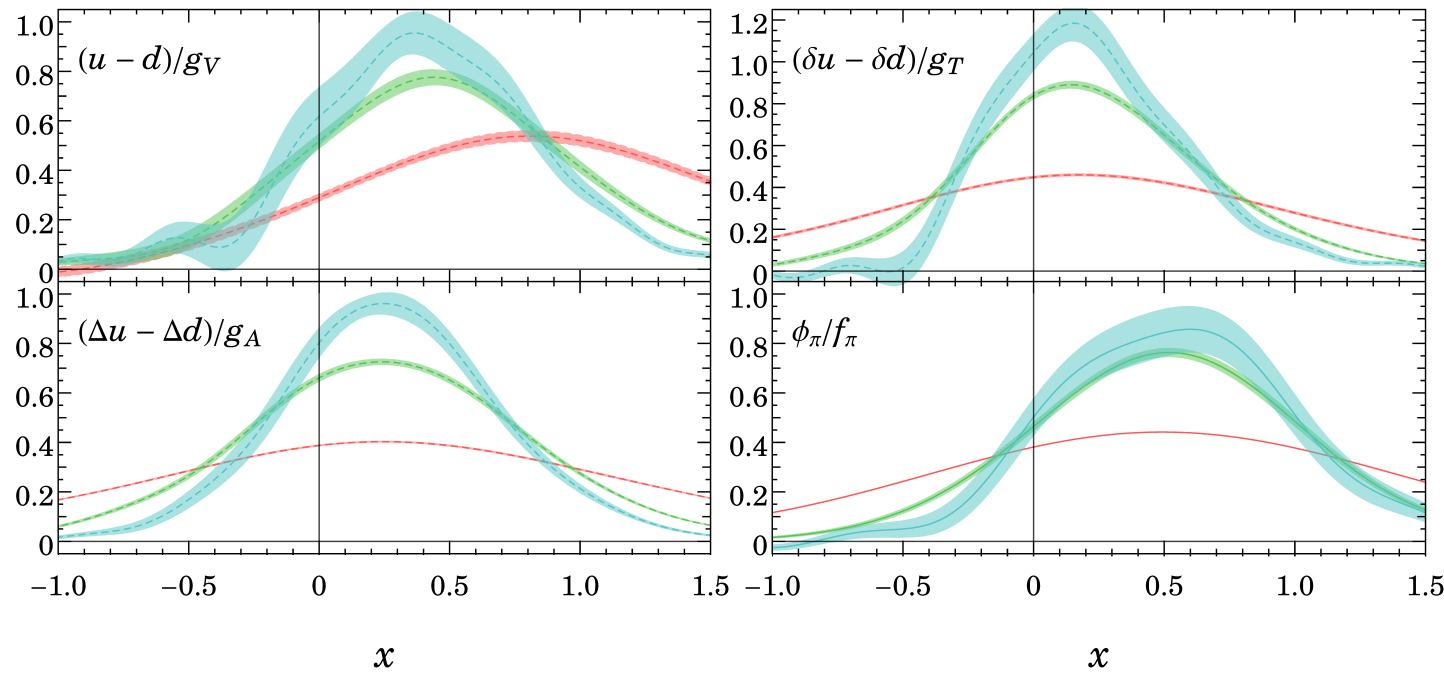

Figure 2: Nucleon isovector quark distributions (upper left), helicity (lower left), transversity (upper right) and pion distribution amplitude (lower right) as functions of $x$, normalized by $g_{V, A, T}$ and $f_{\pi}$, respectively. The different colors indicate $P_{z}$ (in units of $2 \pi / L$ ) 1 (red), 2 (green), 3 (cyan).

refer readers to Ref. [17] and references within.

Lastly, we also observe a large sea asymmetry in the transversity distribution, which is in contradiction with most model calculations. Our $\int_{0}^{\infty} d x(\delta \bar{u}(x)-\delta \bar{d}(x))$ gives $-0.320(18)$. The chiral-quark soliton model combined with large- $N_{c}$ limit gives [18] $\int_{1}^{0} d x(\delta \bar{u}(x)-\delta \bar{d}(x))$ to be -0.082 . This gives agreement in terms of sign but not magnitude, even though the model seems to agree well with our values on the polarized and unpolarized cases. Transversity distributions of sea quarks are often neglected in analyses of experimental parton transversity; our results suggest that this is not a safe assumption.

These techniques are also applicable to the pion distribution amplitude, a key quantity on the light-cone, through

$$
\int \frac{d z}{2 \pi} e^{-i z k_{z}}\left\langle 0\left|\bar{d}(z) \gamma_{z} \gamma_{5} \exp \left(-i g \int_{0}^{z} d z^{\prime} A_{z}\left(z^{\prime}\right)\right) u(0)\right| \pi^{+}(P)\right\rangle .
$$

Hadronic distribution amplitudes are important because they are fundamental nonperturbative inputs for many theoretical tools.Similar to the nucleon case, we also observe that the pion lattice matrix elements have signal extending no more than 10 lattice links. The lower-right panel of Fig. 2 shows the first lattice-QCD calculation extracting this quantity directly ${ }^{2}$. The $0^{\text {th }}$ moment of the distribution gives the pion decay constant $f_{\pi}$, which is consistent an independent measurement on the same ensemble; here, we normalize the distribution with $f_{\pi}$. We see the distribution seems to be converging at the largest two momenta; this is expected, since the pion mass is significantly smaller than the nucleon mass, and thus, has smaller $M_{\pi}^{2} / P_{z}^{2}$ correction. However, there is still significant nonzero distribution in the valence region for $x>1$ and $x<0$, which needs to be corrected

\footnotetext{
${ }^{2}$ An attempt using the lattice-QCD lowest moments to reconstruct the distribution can be found in Ref. [19]
} 
with upcoming matching to infinite-momentum frame. One interesting feature is that the largest momentum distribution peaks around $x=0.5$ as anticipated in QCD models.

There are several improvements that can be done to further refine the current calculations, such as reducing the quark mass to physical, increasing the number of measurements to reduce statistical error, using finer lattice spacing to accommodate larger boosted momenta and improve resolution and using larger lattice volumes to access small $x$. Our current calculation is done using 310$\mathrm{MeV}$ pions, so we anticipate that the distribution will differ quantitatively from the current global analyses, especially on the valence-quark distribution, which is well constrained by data. However, we hope to bring some insight to the qualitative sea asymmetry, about which assumptions must often be made (especially in the polarized case) in the global analyses. From the lattice structures, the leading moments (charges, $\langle x\rangle$, etc.) do not differ by more than a factor of 2 between our current quark mass and the physical one. The sign and the order-of-magnitude of the antiquark asymmetry in nucleon at the largest momentum we have gives some insight into this poorly known territory. The removal of the leading mass correction to all orders and matching from finite momentum to the infinite-momentum frame will be implemented in the near future.

\section{References}

[1] R. G. Edwards and B. Joo (SciDAC Collaboration, LHPC Collaboration, UKQCD Collaboration), Nucl.Phys.Proc.Suppl. 140, 832 (2005), hep-lat/0409003.

[2] C.-Y. Chen, P. S. B. Dev, and A. Soni (2013), 1309.1764.

[3] P. Jimenez-Delgado, W. Melnitchouk, and J. Owens, J.Phys. G40, 093102 (2013), 1306.6515.

[4] U. Aglietti, M. Ciuchini, G. Corbo, E. Franco, G. Martinelli, et al., Phys.Lett. B432, 411 (1998), hep-ph/9804416.

[5] K.-F. Liu, Phys.Rev. D62, 074501 (2000), hep-ph/9910306.

[6] W. Detmold and C. D. Lin, Phys.Rev. D73, 014501 (2006), hep-lat/0507007.

[7] W. Detmold, W. Melnitchouk, and A. W. Thomas, Phys.Rev. D68, 034025 (2003), hep-lat/0303015.

[8] X. Ji, Phys.Rev.Lett. 110, 262002 (2013), 1305.1539.

[9] A. Bazavov et al. (MILC Collaboration), Phys.Rev. D87(5), 054505 (2013), 1212.4768.

[10] T. Bhattacharya, S. D. Cohen, R. Gupta, A. Joseph, and H.-W. Lin (2013), 1306.5435.

[11] M. Arneodo et al. (New Muon Collaboration), Phys.Rev. D50, 1 (1994).

[12] M. Adams et al. (E665 Collaboration), Phys.Rev.Lett. 75, 1466 (1995).

[13] R. Towell et al. (NuSea Collaboration), Phys.Rev. D64, 052002 (2001), hep-ex/0103030.

[14] K. Ackerstaff et al. (HERMES Collaboration), Phys.Rev.Lett. 81, 5519 (1998), hep-ex/9807013.

[15] E. Aschenauer, A. Bazilevsky, K. Boyle, K. Eyser, R. Fatemi, et al. (2013), 1304.0079.

[16] A. Airapetian et al. (HERMES Collaboration), Phys.Rev. D71, 012003 (2005), hep-ex/0407032.

[17] J.-C. Peng, Eur.Phys.J. A18, 395 (2003), hep-ph/0301053.

[18] B. Dressler, K. Goeke, P. Pobylitsa, M. V. Polyakov, T. Watabe, et al. (1998), hep-ph/9809487.

[19] I. Cloet, L. Chang, C. Roberts, S. Schmidt, and P. Tandy, Phys.Rev.Lett. 111, 092001 (2013), 1306.2645. 